\title{
Boundary integral method for Stokes flow with linear slip flow conditions in curved surfaces
}

\author{
C. Nieto, M. Giraldo \& H. Power \\ Instituto de Energía y Termodinámica, \\ Universidad Pontificia Bolivariana, Colombia \\ School of Mechanical, Materials and Manufacturing Engineering, \\ The University of Nottingham, $U K$
}

\begin{abstract}
The no slip boundary condition is traditionally used to predict velocity fields in macro scale flows. When the scale of the problem is about the size of the mean free path of particles, it is necessary to consider that the flow slips over the solid surfaces and the boundary condition must be changed to improve the description of the flow behaviour with continuous governing fluid flow equations. Navier's slip boundary condition states that the relative velocity of the fluid respect to the wall is directly proportionally to the local tangential shear stress. The proportionally constant is called the slip length, which represent the hypothetical distance at the wall needed to satisfy the condition of no-slip flow. Some works have misused boundary conditions derived from Navier's work to model slip flow behaviour for example by employing expressions, for diagonal and curved surfaces, that were derived for flat infinite surfaces aligned with coordinate axes. In this work, the creeping flow of a Newtonian fluid under linear slip conditions is simulated for the cases of a Slit and a Couette mixer by means of the Boundary Element Method (BEM). In the evaluation of such flows, different magnitudes of slip length from 0 (no slip) to 1.0 are analysed in an effort to understand the effect of the slip boundary condition on the physical behaviour of the simulation system. Analytic solutions for both geometries under slip flow are used to estimate $L 2$ norm error, which is below $0.25 \%$ for Couette flow and $1.25 \%$ for Slit flow, validating the approximation applied.

Keywords: slip flow, linear slip boundary conditions, boundary integral methods, Couette mixer.
\end{abstract}




\section{Introduction}

Microsystems Technology (MST) devices are widely used in life sciences and chemistry applications, and its potential uses extend to medical sample testing [1] and drug delivery systems [2]; gas and liquid heat exchangers [3,4] and chemical mixers [5] for enhancement of heat and mass transfer rates; and fluid control and measurement [6] devices. Micro heat exchangers and mixers are currently used for steam gas reforming to produce alternative fuels [7], nuclear resources exploiting [8], micro integrated circuits cooling [9], micro fuel cells [10], among others.

When geometry devices are scaled down, the surface-to-volume ratio increases dramatically so that the surface related phenomena become increasingly dominant, e.g. micro heat exchangers and micro mixers present higher heat and mass transfer rates than macro systems of equal capacity [11]. Therefore, some new features emerges when mechanical structures are sufficiently small, and it becomes important to understand the various types of interactions that arises between the fluid flow constituents and the solid surfaces that contain it.

Different phenomena associated with surface-fluid interactions can be expected when the continuum assumption is close to being broken. For gases, four important effects appear: rarefaction, compressibility, viscous heating and thermal creep. In liquids, phenomena like wetting, adsorption and electrokinetics may be present [12]. However, in both liquids and gases, a phenomenon known as the slip flow regime emerges as a consequence of an insufficient number of molecules in the sampling region [13], affecting the momentum transport at solid-fluid interfaces compared with no slip type flows (i.e. macro scale flows).

So far, micro fluid flow behaviour has been studied under continuum [14] as well molecular approaches $[15,16]$, with the aim of characterize and optimize the operation of MST systems. In order for a fluid to be modelled as a continuum, all of its properties (i.e. kinematic, transport and thermodynamics properties) must be continuous; for that to be possible, enough molecules must be included compared to the length scale of the flow. In the case of gases, this premise is satisfied when the length scale based on transport properties is greater than $1 \mu \mathrm{m}\left(10^{-6} \mathrm{~m}\right)$; for liquids the length scale is based on transport properties and must be larger than $10 \mathrm{~nm}\left(10^{-8} \mathrm{~m}\right)$ [17]. Appropriate velocity slip and temperature conditions at the wall surface must be used to employ continuum models to describe flow behaviour in microflow devices. Navier's slip boundary condition states that the relative tangential fluid velocity, $u_{t}^{f}$, with relation to wall velocity, $U_{t}^{w}$, is directly proportionally to the local shear rate projection in the tangential direction, $\dot{\gamma}_{t}$, as presented in equation (1). The proportionally constant is called slip length $L_{s}$, and represent the hypothetical distance at the wall needed to satisfy the no-slip flow condition [17].

$$
u_{t}^{f}-U_{t}^{w}=L_{s} \dot{\gamma}_{t}
$$

The main difficulty present when applying the previous boundary condition is related to evaluation of tangential shear rate at solid-fluid interface. Linear boundary slip conditions have been applied to predict microflow behaviour in plane 
geometries with continuum governing equations. Attempts to apply this type of boundary conditions for curved surfaces have conducted to inappropriate microflow results due to mistreatment of mathematical models (see [18] for a compilation of those works). Boundary Integral Methods (BIM) relate boundary values for velocity and traction into the integral equations, rather than values throughout the domain like in other partial differential equation numerical solution schemes. Evaluation of slip fluid flow behaviour with BIM can be done by expressing tangential shear rate present in the slip boundary condition (1) in terms of surface tractions at boundaries. This allows a more efficient and easy to use numerical evaluation scheme to analyse micro fluid flow under slip flow regime.

Luo and Pozrikidis [19] study the motion of spherical particles in infinite fluid and near a plane wall subjected to slip boundary conditions. The boundary integral formulation presented in this work takes advantage of the axial symmetry of the boundaries with respect to the axis that is normal to the wall and passes through the particle center, reducing the solution to a system of one-dimensional integral equations. The previous system of equations is valid for sphere and the zero-thickness disk, since the axisymmetry is lost as these particles tumble under the influence of a shear flow. Results for torque and drag over sphere show reduction associated to slip condition at those scales demonstrated the validity of numerical values when compared with analytic results.

The objective of this paper is to present an implementation of a direct BIM to solve Stokes equations under slip boundary conditions for curved geometries. The tangential shear rate at solid-fluid interfaces is evaluated in terms of the surface traction; producing accurate results for fluid flow behaviour in plane and curved geometries not subjected to symmetry conditions. This will allow the evaluation of more complex geometries such as present in micro-scale devices, like $\mathrm{T}$ type mixers, micro-finned heat exchangers, control valves, among others. Analytic solutions for Couette and Slit flow are used to test the numerical results obtained when taking into consideration different types of boundary conditions: the first has only Robin boundary conditions while the second is a mixed boundary condition problem (Robin, Dirichlet and Neumann).

This paper is divided as follows. Governing equations for Stokes flow are presented in Section 2. The next section shows integral representation for governing equations presenting slip boundary conditions which are defined in terms of surface traction at the collocation points. Then the numerical scheme used to solve the set of equations and boundary conditions is presented. Numerical results for Couette and Slit flow are compared with analytic results through evaluation of $L 2$ norm error. Finally, conclusions regarding physical and mathematical considerations are given.

\section{Governing equations}

Fluid flow in micro scale devices usually happens at very low Reynolds number due to the magnitudes of velocities and characteristic lengths are small enough compared to fluid density and viscosity. In these cases, fluid flow can be mod- 
elled by application of the Stokes system of equations (2), which states a balance between the pressure force in the fluid and the viscous-shear force at all points in the fluid.

$$
\frac{\partial u_{i}}{\partial x_{i}}=0 \quad \frac{\partial \sigma_{i j}}{\partial x_{j}}=0
$$

where

$$
\sigma_{i j}=-p \delta_{i j}+\mu\left(\frac{\partial u_{i}}{\partial x_{j}}+\frac{\partial u_{j}}{\partial x_{i}}\right)
$$

In equations (2)-(3), $\vec{u}$ is the velocity, $p$ the pressure, $\delta_{i j}$ the Kronecker delta, and $\mu$ is the viscosity of the fluid.

Boundary conditions are defined depending to the problem that is being solved. In the case of Couette flow (or the flow between concentric cylinders), the external cylinder is stationary while the internal one rotates at a constant angular dimensionless velocity of value 1. For Slit flow (or fully developed flow between parallel plates), the superior and inferior surfaces are stationary, while at the entrance and exit the perpendicular velocities are made zero and the tractions are given only by a pressure difference between them. For the cases shown dimensionless pressure, $\Delta p$ equal to 1 is considered. Slip behaviour defined in equation (1) is considered by expressing local tangential shear rate in terms of vector surface traction defined in (4). Additionally, variation in momentum transport at solid-fluid interface is analysed by evaluation of slip length $L_{s}$ between zero (no slip condition) and 1.0.

\section{Boundary integral formulation for slip flow regime}

The Stokes velocity field has the following direct integral representation formulae for an arbitrary point $x$ in a closed domain $\Omega_{i}$ filled with a Newtonian fluid [20]:

$$
c_{i} u_{i}(x)-\int_{S} K_{i j}(x, y) u_{j}(y) d S_{y}+\int_{S} u_{i}^{j}(x, y) f_{j}(y) d S_{y}=0
$$

where $f$ is the vector surface tractions $\left(f_{j}(y)=\vec{\sigma}_{i j}(\vec{u}, p) n_{j}\right)$, and $c$ is a constant dependent on the position of the source point. For internal points $c=1$ and for point at a smooth boundary $c=1 / 2$. The Stokeslet and the corresponding surface traction or Stresslet for two dimensions are given by:

$$
\begin{gathered}
u_{i}^{j}(x, y)=-\frac{1}{4 \pi}\left[\ln \left(\frac{1}{r}\right) \delta_{i j}+\frac{\left(x_{i}-y_{i}\right)\left(x_{j}-y_{j}\right)}{r^{2}}\right] \\
K_{i j}(x, y)=-\frac{1}{\pi} \frac{\left(x_{i}-y_{i}\right)\left(x_{j}-y_{j}\right)\left(x_{k}-y_{k}\right) n_{k}(y)}{r^{4}}
\end{gathered}
$$

being $r$ the Euclidean distance between point $x$ and $y, r=|x-y|$. 
The tangential projection of the surface traction can be expressed as follows:

$$
\begin{aligned}
f_{i} s_{i} & =\sigma_{i j} n_{j} s_{i}=\left[-p n_{i} s_{i}+\mu\left(\frac{\partial u_{i}}{\partial x_{j}}+\frac{\partial u_{j}}{\partial x_{i}}\right) n_{j} s_{i}\right] \\
& =\mu\left(\frac{\partial u_{i}}{\partial x_{j}}+\frac{\partial u_{j}}{\partial x_{i}}\right) n_{j} s_{i}
\end{aligned}
$$

Thus, the slip boundary condition in tangential direction can be expressed in terms of the tangential projection of the surface traction in the following form:

$$
u_{t}^{f}-U_{t}^{w}=L_{s} f_{i} s_{i}=L_{s} \dot{\gamma}_{t}
$$

due to the local shear rate projection in the tangential direction, $\dot{\gamma}_{t}$ is defined as:

$$
\dot{\gamma}_{t}=\left(\frac{\partial u_{i}}{\partial x_{j}}+\frac{\partial u_{j}}{\partial x_{i}}\right) n_{j} s_{i}
$$

where $s_{i}$ is the tangential vector to the boundary surface. The tangential and normal components of fluid velocity can be expressed as:

$$
u_{t}^{f}=u_{i} s_{i} \quad u_{n}^{f}=u_{i} n_{i}
$$

Given the dependency of fluid velocity with nodal values of surface traction at the boundaries, a new system of equations will be defined to simultaneously consider this effect. After discritization using the BIM, equations (4), (8), and (10) can be written in matrix form as:

$$
\begin{gathered}
{[H][\vec{u}]=[G][\vec{f}]} \\
{[S][\vec{u}]-L_{s}[S][\vec{f}]=\left[\overrightarrow{U_{t}^{w}}\right]} \\
{[N][\vec{u}]=\left[\overrightarrow{U_{n}^{w}}\right]}
\end{gathered}
$$

$[H]$ and $[G]$ being the usual matrices in BEM, $[\vec{u}]$ and $[\vec{f}]$ are, respectively, the velocity and surface traction vectors from the boundary problem, and $[S]$ and $[N]$ are matrices for each element built from the tangential and normal vectors in the following arrangements.

$$
\begin{aligned}
& {[S]=\left[\begin{array}{ccccccc}
s_{1}^{1} & s_{2}^{1} & 0 & 0 & 0 & 0 & \cdots \\
0 & 0 & s_{1}^{2} & s_{2}^{2} & 0 & 0 & \cdots \\
0 & 0 & 0 & 0 & s_{1}^{3} & s_{2}^{3} & \cdots \\
\vdots & \vdots & \vdots & \vdots & \vdots & \vdots & \ddots
\end{array}\right]} \\
& {[N]=\left[\begin{array}{ccccccc}
n_{1}^{1} & n_{2}^{1} & 0 & 0 & 0 & 0 & \cdots \\
0 & 0 & n_{1}^{2} & n_{2}^{2} & 0 & 0 & \cdots \\
0 & 0 & 0 & 0 & n_{1}^{3} & n_{2}^{3} & \cdots \\
\vdots & \vdots & \vdots & \vdots & \vdots & \vdots & \ddots
\end{array}\right]}
\end{aligned}
$$


The super index in the previous array represents the node number. The new system of equations is then defined in next form:

$$
[A][\vec{X}]=[\vec{b}]
$$

where new matrices and vectors are defined as (dropping all [ ] ):

$$
\begin{gathered}
{[A]=\left[\begin{array}{cc}
H & -G \\
S & -L_{s} S \\
N & 0
\end{array}\right]} \\
{[\vec{b}]=\left[\begin{array}{c}
0 \\
\vec{U}_{t} \\
\vec{U}_{n}
\end{array}\right]} \\
\vec{X}=\left[\begin{array}{c}
\vec{u} \\
\vec{f}
\end{array}\right]
\end{gathered}
$$

The solution of this complete system of equations conduces to the evaluation of velocities and tractions at the boundaries taking into account the effect of slip conditions over flow behaviour and was solved by simple Gauss elimination method.

\section{Integral equation discretization}

Geometry discretization for numerical integration will be made with quadratic elements with the aim to improve evaluation of velocity and surface tractions at boundaries and to produce a more reliable micro scale flow behaviour. The interpolation scheme for a function $X(\varepsilon)$ is given by:

$$
X(\varepsilon)=\psi_{1}(\varepsilon) X^{(1)}+\psi_{2}(\varepsilon) X^{(2)}+\psi_{3}(\varepsilon) X^{(3)}
$$

where $X^{(1)}, X^{(2)}, X^{(3)}$ are the values of $X(\xi)$ on the three nodes of the element and the interpolation functions $\vec{\psi}$ are as follows:

$$
\psi_{1}=\frac{1}{2} \varepsilon(\varepsilon-1) ; \quad \psi_{2}=(1-\varepsilon)(1+\varepsilon) ; \quad \psi_{3}=\frac{1}{2} \varepsilon(\varepsilon+1)
$$

Standard Guassian Quadrature is used to evaluate the final set of integrals resulting from applying the interpolation function (20). Telles' Transformation [21] and Rigid Body Motion [22] are used to avoid singularity present when integrating over the same element where the source point is located.

\section{Numerical results}

In this section the test performed for the direct boundary integral formulation implemented to predict slip behaviour for Couette and Slit flow is presented, taking into account different momentum transport at the solid-fluid interface by variations of the slip length condition. 


\subsection{Couette flow}

An analytic solution for the tangential fluid velocity in Couette flow under linear slip boundary conditions is given by ( [18]):

$$
u_{t}=\frac{\omega}{A-B}\left(A r-\frac{1}{r}\right)
$$

where

$$
A=1-\frac{2 \frac{L_{s}}{r_{e}}}{r_{e}^{2}} ; \quad B=1-\frac{2 \frac{L_{s}}{r_{i}}}{r_{i}^{2}}
$$

and being $\omega, r_{e}$ and $r_{i}$, the angular velocity for internal cylinder, external and internal radius, respectively. Tangential velocity $u_{t}$ is given for any radius $r$. The slip length $L_{s}$ variates between zero (no slip condition) and 1.0 to account for momentum transport variation at both the internal and external cylinders. When $L_{s}$ is dropped to zero in equation (22), it reduces to Couette flow for no slip conditions as is presented in [23]. A regular mesh was used to solve the linear system of equations: 48 quadratic elements on both boundary surfaces and 300 uniformly distributed internal points. It is important to point out that these internal nodes are not required for the solution of the problem, but only for flow visualization and numerical error evaluation.

Results for both no slip and slip Couette flow are presented in Table 1. Momentum transport modification at the boundaries is evidenced by the reduction in fluid velocities near the internal cylinder ( $70 \%$ for the higher slip length evaluated respect to the no slip condition) as shown in Figure 1(A). For the outer cylinder, the slip regimen induce a velocity over this boundary, which tends to reduce as the slip length is increased, showing the continuity in transport momentum through the fluid flow.

Errors below $0.25 \%$ indicate the good performance of direct boundary integral formulation implemented in the system of equations (10). It is also observed that there is a considerable reduction in error as the slip length is increased in spite of discontinuity in the velocity at the wall and the fluid imposed by the variation in this value. This could be explained by reduction in momentum transferred by the surfaces to the fluid which implies an overall reduction in flow velocity.

Table 1: Assessment of Couette flow under slip conditions.

\begin{tabular}{lccccc}
\hline$L_{s}$ & 0 & 0.1 & 0.25 & 0.5 & 1.0 \\
\hline$L 2$ norm $(\%)$ & 0.248 & 0.252 & 0.175 & 0.127 & 0.063 \\
\hline
\end{tabular}




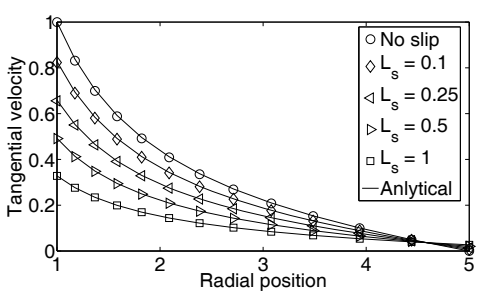

(A)

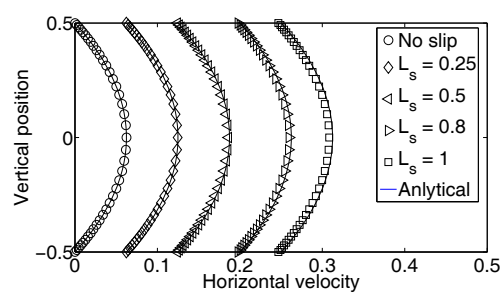

(B)

Figure 1: Velocity profiles for slip (A) Couette and (B) slit flow.

\subsection{Slit flow}

The analytic solution for velocity profile under slip flow over the horizontal surfaces is available in [24]:

$$
u_{1}=\frac{h^{2}}{2 \mu} \frac{\Delta P}{L}\left[1-\left(\frac{x_{2}}{h}\right)^{2}\right]+\frac{h}{\mu} \frac{\Delta P}{L} L_{s}
$$

where $L$ is the channel length, $h$ its height and $\Delta P$ the imposed pressure difference. The second term in the right side of equation (24) accounts the slip effect in the velocity profile. It reduces to no slip when $L_{s}$ is dropped to zero as presented in [23]. The tested mesh consisted of 160 quadratic elements on the outer surface refined at a the corners and 600 internal collocation points.

The results for this case shown an error below to $1.2 \%$, showing a good description of fluid flow for this geometry as can seen in Figure 1(B). An increase in fluid flow velocities is observed with respect to the no slip conditions indicating the effect of slip flow over momentum transport at boundaries. A detailed observation of results in Figure 1(B) and Table 2 shows that deviation from analytic results increase as flow goes from no slip to slip behaviour. Despite the mesh refinement applied at the corners, numerical evaluation of normal and tangential vectors at this positions affects the interior flow description. Furthermore, since the channel has a height equal to the maximum slip length evaluated, an increase over this limit can conduce to unrealistic results according to the definition of slip length in boundary condition (1).

Table 2: Assessment of Slit flow under slip conditions.

\begin{tabular}{lccccc}
\hline$L_{s}$ & 0 & 0.1 & 0.25 & 0.5 & 1.0 \\
\hline$L 2$ norm $(\%)$ & 0.009 & 0.252 & 0.557 & 0.931 & 1.182 \\
\hline
\end{tabular}




\section{Conclusions}

A direct boundary integral method was used to evaluate slip flow behaviour for two traditional fluid flow problems. Results for both problems presented accurate results when compared with analytic results. As expected, a reduction in momentum transportation occurs at the fluid-solid interfaces due to slip regimen, leading to a change in the flow behaviour. A modified system of equations was used to evaluate slip flow in terms of surface traction and the usual matrices in BEM. Despite the system of equations increased from $N$ to $2 N$, the computational effort is kept at low levels, which permits its application to the solution of more complex flow systems.

\section{Acknowledgements}

C. Nieto is supported in his $\mathrm{PhD}$ program by the Universidad Pontificia Bolivariana and COLCIENCIAS under the Convocatoria Banco de Proyectos Tesis Doctorales.

\section{References}

[1] Gad-el Hak, M., MEMS: Applications. CRC Press: Virginia, pp. 11-20, 2006.

[2] C. Kleinstreuer, J.L. \& Koo, J., Microfluidics of nano-drug delivery. International Journal of Heat and Mass Transfer, 51(1), pp. 5590-5597, 2008.

[3] L. Shui, J.E. \& van den Berg, A., Multiphase flow in micro- and nanochannels. Sensors and Actuators B: Chemical, 121(1), pp. 263-276, 2007.

[4] M. Giraldo, Y.D. \& Williams, R., Boundary integral study of nanoparticle flow behaviour in the proximity of a solid wall. Journal of Physics D: Applied Physics, 41(8), pp. 1-10, 2008.

[5] D. Bothe, C.S. \& Warnecke, H.J., Fluid mixing in a t-shaped micro-mixer. Chemical Engineering Science, 61(9), pp. 2950-2958, 2006.

[6] J. Alvarado, J.M. \& Soriano, G., Development and characterization of a capacitance-based microscale flowmeter. Flow Measurement and Instrumentation, 20(2), pp. 81-84, 2009.

[7] A.Y. Tonkovich, Y.W.D.Q.T.L.P., S. Perrya \& Rogersa, W.A., Microchannel process technology for compact methane steam reforming. Chemical Engineering Science, 59(22-23), pp. 4808-4817, 2004.

[8] X. Li, R.L.P. \& Dewson, S.J., Heat exchangers for the next generation of nuclear reactors. Proc. of International Congress on Advances in Nuclear Power Plants, eds. R.R.M.K. F. L. Bowman, S. Anghaie \& A. Rao, ICAPP: Reno, pp. 201-209, 2006.

[9] H. Lee, J.S.J.B.M.K., Y. Jeong \& Chun, K., Package embedded heat exchangernext term for stacked multi-previous termchipnext term module. Sensors and Actuators A: Physical, 114(2-3), pp. 204-211, 2004.

[10] S. Won Cha, R.O. \& Prinz, F.B., The influence of size scale on the performance of fuel cells. Solid State Ionics, 175(1-4), pp. 789-795, 2004. 
[11] Hu, G. \& Li, D., Multiscale phenomena in microfluidics and nanofluidics. Chemical Engineering Science, 62(13), pp. 3443-3454, 2007.

[12] G. Karniadakis, A.B. \& Aluru, N., Microflows and Nanoflows: Fundamentals and Simulation. Springer: New York, p. 487, 2005.

[13] Thompson, P.A. \& Troian, S.M., A general boundary condition for liquid flow at solid surfaces. Nature, 389(1), pp. 360-362, 1997.

[14] Gad-el Hak, M., Gas and liquid transport at the microscale. Heat Transfer Engineering, 27(4), pp. 1-17, 2006.

[15] Bird, G., Molecular gas dynamics and direct simulation of gas flows. Oxford University Press: Oxford, p. 484, 1994.

[16] Sadus, R.J., Molecular simulation of fluids: Theory, applications and objectorientation. Elsevier: Amsterdam, p. 562, 1999.

[17] Nguyen, N.T. \& Wereley, S.T., Fundamentals and Applications of Microfluidics. Artech House: Norwood, p. 487, 2006.

[18] R. W. Barber, X.J.G., Y. Sun \& Emerson, D.R., Isothermal slip flow over curved surfaces. Vacuum, 76(1), pp. 73-81, 2004.

[19] Luo, H. \& Pozrikidis, C., Effect of surface slip on stokes flow past a spherical particle in infinite fluid and near a plane wall. Journal of Engineering Mathematics, 62(1), pp. 1-21, 2008.

[20] Power, H. \& Wrobel, L., Boundary Integral Methods in Fluid Mechanics. Computational Mechanics publications: Southhampton, pp. 147-194, 1995.

[21] Telles, J., A self-adaptative coordinate transformation for efficient numerical evaluation of general boundary element integrals. International Journal for Numerical Methods in Engineering, 24(1), pp. 959-973, 1987.

[22] Brebbia, C. \& Dominguez, J., Boundary Elements: An Introductory Course. Computationanl Mechanics Publications: Southampton, p. 400, 1992.

[23] Currie, I., Fundamental Mechanics of Fluids. Macerl Dekker: New York, pp. 259-262, 2003.

[24] Matthews, M.T. \& Hill, J.M., Newtonian flow with nonlinear navier boundary condition. Acta Mechanica, 191(1), pp. 195-217, 2007. 